\title{
ELEMENTARY PROPERTIES OF ORDERED ABELIAN GROUPS
}

\author{
BY \\ ABRAHAM ROBINSON AND ELIAS ZAKON
}

Introduction. A complete classification of abelian groups by their elementary properties (i.e. properties that can be formalized in the lower predicate calculus) was given by Szmielew [9]. No such attempt, however, has so far been made with respect to ordered groups. In the present paper the classification by elementary properties will be carried out for all archimedean ordered abelian groups and, moreover, for a certain more general class of groups which we shall call regularly ordered. Simultaneously, necessary and sufficient conditions will be established for two such groups to be elementarily equivalent, i.e. to have all their elementary properties in common (see Theorem 4.7).

By a complete classification of a class of groups we mean its partition into disjoint subclasses in such a way that two groups belong to one subclass if, and only if, they are elementarily equivalent. This goal will be attained by setting up a series of (finite or infinite) complete systems of axioms, each system defining a certain subclass of ordered abelian groups.

The notation and terminology of [8] will be used throughout. In particular, the concept of model-completeness introduced in [8] and [7] will be applied. A novelty feature of the present paper is that the method based on model-completeness will be combined with what we shall call "adjunction of new relations." To illustrate the usefulness of the method, we shall also apply it to give new simplified proofs of some theorems by Langford and Tarski on the completeness of certain systems of axioms referring to ordered sets. This will constitute an additional result of the paper (see $\$ 2$ ).

1. Preliminaries, terminology and notation. The concept of an ordered set can be formalized in the lower predicate calculus by means of the following system of axioms based on a binary relation of "equivalence," $E(x, y)$, (read: " $x$ is equivalent to $y$ ") and a binary relation of "order," $Q(x, y)$ (read: " $x$ is less than, or equivalent to, $\left.y^{\prime \prime}\right)$ :

\subsection{Axioms of Equivalence $\left({ }^{1}\right)$.}

(a) $(x) E(x, x)$.

(b) $(x)(y)[E(x, y) \cdot \supset \cdot E(y, x)]$.

(c) $(x)(y)(z)[E(x, y) \wedge E(y, z) \cdot \supset \cdot E(x, z)]$.

(d) $(\exists x)(\exists y)[\sim E(x, y)]$.

Received by the editors July 20, 1959.

(1) We use the connectives $\wedge, \vee, \sim, \supset \cdot, \cdot \equiv \cdot$ for conjunction, disjunction, negation, implication and logical equivalence respectively. $(x)$ is the universal and $(\exists x)$ the existential quantifier. 


\subsection{AXIOMS OF ORDER.}

(a) $(x)(y)[Q(x, y) \wedge Q(y, x) \cdot \equiv \cdot E(x, y)]$.

(b) $(x)(y)[Q(x, y) \vee Q(y, x)]$.

(c) $(x)(y)(z)[Q(x, y) \wedge Q(y, z) \cdot \supset \cdot Q(x, z)]$.

(d) $(x)(y)(z)(w)[Q(x, y) \wedge E(x, z) \wedge E(y, w) \cdot \supset \cdot Q(z, w)]$.

To obtain the concept of an ordered abelian group we add a ternary relation, $S(x, y, z)$, (read: " $z$ is the sum of $x$ and $y$ ") and the following axioms:

\subsection{GROUP AXIOMS $\left({ }^{2}\right)$.}

(a) $(x)(y)(\exists z) S(x, y, z)$.

(b) $(x)(y)(z)(w)[S(x, y, z) \wedge S(x, y, w) \cdot \supset \cdot E(z, w)]$.

(c) $(x)(y)(z)[S(x, y, z) \cdot \supset \cdot S(y, x, z)]$.

(d) $(u)(v)(w)(x)(y)(z)[S(u, v, w) \wedge S(w, x, y) \wedge S(v, x, z) \cdot \supset \cdot S(u, z, y)]$.

(e) $(u)(v)(w)(x)(y)(z)[S(u, v, w) \wedge E(u, x) \wedge E(v, y) \wedge E(w, z) \cdot \supset \cdot S(x, y, z)]$.

(f) $(x)(y)(\exists z) S(x, z, y)$.

(g) $(x)(y)(z)(v)(w)[Q(y, v) \wedge S(x, y, z) \wedge S(x, v, w) \cdot \supset \cdot Q(z, w)]$.

The three predicates, $E, Q, S$, will be called the "atomic predicates" of our system of axioms. Any well-formed formula of the lower predicate calculus that does not contain any predicates except $E, Q, S$ (or is logically equivalent to such a formula) will be referred to as an elementary formula of group theory. If ordered sets (not groups) are considered, the same definitions apply, with the only difference that the relation $S$ is excluded $\left({ }^{3}\right)$. A set of statements $K$ (in particular, a set of axioms) is said to be elementary if so are all the statements belonging to $K . K$ is referred to as complete if, for any elementary statement $X$ which does not contain any constants other than those occurring in $K$, either $X$ or $\sim X$ is deducible from $K$.

A formula is said to be atomic if it consists of a single atomic predicate in which the argument places have been filled by individual variables or constants; in our case then all atomic formulae are of the form $E(x, y)$ or $Q(x, y)$ or $S(x, y, z)$. A well-formed formula is referred to as primitive if it has the form

$$
\left(\exists y_{1}\right)\left(\exists y_{2}\right) \cdots\left(\exists y_{n}\right) Z\left(y_{1}, y_{2}, \cdots, y_{n}\right)
$$

where $Z\left(y_{1}, \cdots, y_{n}\right)$ is a conjunction of atomic formulae and (or) their negations, which contain the free variables $y_{1}, y_{2}, \cdots, y_{n}$. For example, we

(2) In 1.3 the axioms (a), (b) express the fact that the group is closed under addition and that the sum is unique; axioms (c), (d) give the commutative and associative laws; (e) expresses the substitutivity of the equivalence relation with respect to addition; (f) ensures the existence of the inverse, and $(\mathrm{g})$ means, in ordinary notation, that $y \leqq v$ always implies $x+y$ $\leqq x+v$.

(3) For the general concept of an elementary formula, cf. [8]. 
shall define a sequence of primitive formulae, $D_{n}(x)(n=2,3,4, \cdots)$ that will be used later $\left({ }^{4}\right)$ :

$$
D_{n}(x) \cdot \equiv \cdot\left(\exists y_{1}\right)\left(\exists y_{2}\right) \cdots\left(\exists y_{n-1}\right)\left[S\left(y_{1}, y_{1}, y_{2}\right) \wedge S\left(y_{1}, y_{2}, y_{3}\right) \wedge \cdots\right.
$$

$$
\left.\wedge S\left(y_{1}, y_{n-2}, y_{n-1}\right) \wedge S\left(y_{1}, y_{n-1}, x\right)\right]
$$

Now let $K$ be a consistent elementary set of axioms, $M$ a model of $K$, and $X$ some elementary statement; then $X$ is said to be defined in $K$ (in $M$ respectively) if $X$ does not contain any constants other than those occurring in $K$ (denoting elements of $M$, respectively). Any other model $M^{\prime}$ of $K$ is called an extension of $M$ if $M \subseteq M^{\prime}$ and if every atomic statement defined in $M$ holds either in both $M$ and $M^{\prime}$ or in none of them. $M$ is called a prime model of $K$ if every other model $M^{\prime}$ of $K$ contains a model $M^{\prime \prime}$ of $K$ such that $M^{\prime}$ is an extension of $M^{\prime \prime}$, and $M^{\prime \prime}$ is isomorphic to $M$.

We shall now list, for further references, some theorems proved in [8] that will be needed in the sequel. (The first of them can serve as a definition of the important concept of model-completeness):

1.6. A consistent elementary set of axioms $K$ is model-complete if, and only if, for every pair of models of $K, M$ and $M^{\prime}$, such that $M^{\prime}$ is an extension of $M$, no primitive statement $Y$ which is defined in $M$ can hold in $M^{\prime}$ unless it holds already in $M$ (cf. [8, p. 16]).

1.7. If a model-complete set of axioms $K$ has a prime model, then $K$ is complete (cf. [8, p. 74]).

1.8. Let $K$ be a model-complete countable set of axioms with the following properties: (a) Any two countable models of $K$, which have no constants in common other than the constants of $K$ (if any), can be embedded in a joint extension $M$; (b) $K$ has infinite models only. Under these assumptions, $K$ is complete $\left(^{5}\right)$.

It should be well noted that all notions defined in this section are dependent on the choice of the atomic predicates (in our case $E, Q$ and $S$ ). Any change in that choice automatically involves a change in the meaning of such terms as "elementary formula," "atomic formula," "primitive formula," "extension," etc. Such a change occurs, in the first place, whenever entirely new atomic predicates (undefinable in terms of the already given ones) are introduced. In this paper, however, we shall be concerned solely with predi-

(4) In ordinary terminology the formula $D_{n}(x)$ means: "the group element $x$ is divisible by the integer $n$," i.e. "there exists an element $z$ such that $n z=x$." Formula 1.5 gives the definition of $D_{n}(x)$ in terms of our atomic predicates. Each $D_{n}$ is a primitive monadic predicate.

(5) This is a modified version of Theorem 4.2 .1 proved in $[8, \mathrm{p} .74]$. It is proved in the same way as the latter, with insignificant changes based on the well known theorem by Loewenheim-Skolem which makes it possible to replace uncountable models by countable ones whenever the set of axioms is countable. The term "countable," in this paper, always means "denumerably infinite." 
cates that can be defined in terms of $E, Q$ and $S$, such as, for instance, the predicates $D_{n}$ defined in $\mathbf{1 . 5}$.

It is very important, for our purposes, to note the simple fact that we may, of course, regard such definable predicates as new atomic ones, provided only that we treat their definitions as new axioms to be added to the originally given system of axioms $K$. In this way a new system of axioms, $K^{*}$ say, is obtained which we shall strictly distinguish from the original system $K$. This distinction is necessary because $K^{*}$ contains new atomic predicates, and, consequently, such concepts as "primitive formula," "prime-model," "extension of a model," etc. obtain a different meaning in $K^{*}$. As we shall see, it may happen that $K^{*}$ is model-complete whereas $K$ is not. On the other hand, it is clear that the consistency and completeness of $K$ implies that of $K^{*}$, and vice versa; for the introduction of well-formed definitions in the capacity of axioms has no effect on either consistency or completeness. This fact will be used later.

The process of adjoining definable predicates in the capacity of new atomic predicates and, simultaneously, adjoining their definitions as new axioms, will be referred to as adjunction of new relations. It will be applied in almost all our proofs.

2. Some completeness theorems on ordered sets $\left({ }^{6}\right)$. In this section the predicate $S$ and Axioms 1.3 will be excluded; so only the predicates $E$ and $Q$ of $\$ 1$ count as atomic. We shall start with Axioms 1.1 and 1.2. This set of axioms is incomplete. It can, however, be made complete by adjoining some new axioms, as will be shown below.

I. Let us adjoin the two axioms:

$$
\begin{gathered}
(x)(y)(\exists z)\{Q(y, x) \vee[\sim Q(z, x) \wedge \sim Q(y, z)]\} . \\
(x)(\exists y)(\exists z)[\sim Q(x, y) \wedge \sim Q(z, x)] .
\end{gathered}
$$

These axioms state, in ordinary language, that the ordered set under consideration is densely ordered (2.1), and that it has no first and no last element (2.2). Now we shall prove the following:

2.3. THEOREM. The system of axioms for a densely ordered set with no first and no last element in it (i.e. the system consisting of 1.1, 1.2, 2.1 and 2.2) is complete.

Proof. First we shall show that the system of these axioms (call it $K$ ) is model-complete. As $K$ is clearly consistent and elementary, we may apply 1.6. Let $M$ be a model of $K, M^{\prime}$ an extension of $M$, and $Y$ a primitive statement of the form 1.4, defined in $M$, and holding in $M^{\prime}$. We have to show

${ }^{(6)}$ Equivalent or similar theorems have been proved by Langford [5] and Tarski [10]. However, the proofs given in this section are considerably simpler than all other proofs known to the authors, due to the application of the theory of model-completeness. 
that $Y$ holds already in $M$. By definition (1.4), the statement $Y$, when translated into ordinary language, means that a particular finite system of equations and inequalities of the type

$$
\alpha=\beta, \quad a \neq \beta, \quad a<\beta, \quad \alpha \nless \beta
$$

possesses a solution (or, as we shall say, is satisfiable) $\left({ }^{7}\right)$. Given that (2.3.1) has a solution in $M^{\prime}$, we have to show that it is satisfiable already in $M$. Without loss of generality, we may simplify the system (2.3.1) by eliminating from it all formulae of the type $\alpha \neq \beta, \alpha \nless \beta$, and $\alpha=\beta$. In fact, a formula of the type $\alpha \neq \beta$ is equivalent to the disjunction $\alpha<\beta \vee \alpha>\beta$, where at least one of the disjuncts must hold in $M^{\prime}$, so that the other one can be dropped without affecting the satisfiability of (2.3.1). Similarly, any formula of the type $\alpha \nless \beta$ splits into $\beta=\alpha \bigvee \beta<\alpha$ where one of the disjuncts can be dropped. When this process is completed we shall be left only with formulas of the type $\alpha=\beta$ and $\alpha<\beta$. Finally, the formulas of the type $\alpha=\beta$ are disposed of by simply dropping them after replacing everywhere " $\beta$ " by " $\alpha$ " (i.e. by eliminating $\beta$ from the system). Thus we may assume that (2.3.1) consists only of inequalities of the type $\alpha<\beta$. Now let $\left(y_{1}, y_{2}, \cdots, y_{n}\right)$ be a solution of that system of inequalities in $M^{\prime}$ (so that $y_{1}, y_{2}, \cdots, y_{n} \in M^{\prime}$ ); further, let $a_{1}, a_{2}, \cdots, a_{m}$ be all the constants of $M$ occurring in (2.3.1). As $M^{\prime}$ is a model of 1.1 and 1.2 , i.e. an ordered set containing all the $y_{i}$ and $a_{j}$, we can arrange the latter in the same order in which they follow each other in $M^{\prime}$, say

$$
y_{1}<y_{2}<a_{1}<\cdots<y_{k}<a_{z}<y_{k+1}<\cdots<a_{m}<y_{n-1}<y_{n} .
$$

Clearly, we shall solve (2.3.1) within $M$ if we succeed in replacing the $y_{i}$ by some elements of $M$ in such a way that the inequalities (2.3.2) are preserved in $M$. As regards the constants $a_{j}$, they follow each other in $M$ in the same order as in $M^{\prime}$ (for $M^{\prime}$ is an extension of $M$; so the atomic statements " $\alpha \leqq \beta$ " hold in $M$ whenever they hold in $M^{\prime}$ ). All we have to do then is to insert between the $a_{j}$ (and, possibly, before or after them) some elements $x_{i} \in M$, in the same way as the $y_{i}$ appear in (2.3.2). This, however, must be possible, for $M$ is a model of $K$, i.e. a densely ordered set with no first and no last element in it. Thus, by 1.6, the model-completeness of $K$ is proved. On the other hand, $K$ clearly has prime models, e.g. the set of all rational numbers in their natural order $\left.{ }^{8}\right)$. Hence, by $1.7, K$ is complete, and our Theorem is proved.

II. Next we shall consider densely ordered sets which have a first but no last element. Such sets can be characterized by a system of axioms consisting of 1.1, 1.2, 2.1 and the following axiom (which replaces 2.2):

(7) The formulae of (2.3.1) only typify the equations (or inequalities) of the system, and every one of the four types may occur a finite number of times. The Greek letters $\alpha, \beta$ stand for constants of $M$ or for the "unknowns," $y_{1}, y_{2}, \cdots, y_{n}$, of the system.

${ }^{(8)}$ For every densely ordered set contains a subset isomorphic to the ordered set of all rationals; cf. [2, p. 60]. 


$$
(\exists x)(y) Q(x, y) \wedge(x)(\exists y)[\sim Q(y, x)] .
$$

Again we can prove this system to be complete:

2.5. THEOREM. The system of axioms for a densely ordered set with a first but no last element in it (i.e. the system consisting of 1.1, 1.2, 2.1 and 2.4) is complete.

Proof. A direct application of (1.6) fails this time, for our system of axioms (call it again $K$ ) is model-incomplete $\left({ }^{9}\right)$. So we first adjoin a new relation, $P(x)$, defined by the axiom $\left({ }^{10}\right)$

$$
P(x) \cdot \equiv \cdot(y) Q(x, y) .
$$

Let $K^{*}$ be the system of axioms consisting of 1.1, 1.2, 2.1, 2.4 and 2.6, with the atomic predicates $E, Q, P$. We assert that $K^{*}$ is model-complete. In fact, let $M$ be a model of $K^{*}, M^{\prime}$ its extension and suppose that a primitive statement $Y$ (see 1.4) is defined in $M$ and holds in $M^{\prime}$. In ordinary language, $Y$ means that a particular finite system of conditions of the types

$$
\alpha=\beta, \alpha \neq \beta, \alpha<\beta, \alpha \nless \beta, P(\alpha), \sim P(\alpha)
$$

is satisfiable. Given that (2.6.1) is satisfiable in $M^{\prime}$, we have to prove the same for $M$. By 2.4, $M$ has a first element, say $a_{0}$. Then the statement $P\left(a_{0}\right)$ holds in both $M$ and $M^{\prime}$ [(for $P\left(a_{0}\right)$ is atomic; so it must hold in every extension of $M]{ }^{(11)}$. Hence, all conditions of the type $P(\alpha)$ and $\sim P(\alpha)$ in (2.6.1) may be replaced by $\alpha=a_{0}$ and $\alpha \neq a_{0}$ respectively, so that (2.6.1) reduces to (2.3.1), and the same process as in the proof of 2.3 leads to the conclusion that $K^{*}$ is model-complete, and also complete in the ordinary sense. But then $K$, too, is complete, and our theorem is proved.

Theorems analogous to 2.3 and 2.5 can also be proved, in a similar way, for densely ordered sets with a last but no first element, and for those with both a last and a first element. In particular, in this latter case, we have to replace Axiom 2.4 by

$$
(\exists x)(y) Q(x, y) \wedge(\exists x)(y) Q(y, x)
$$

and to adjoin, besides $P(x)$, an additional new relation $P^{\prime}(x)$, defined by the axiom

$$
P^{\prime}(x) \cdot \equiv \cdot(y) Q(y, x) .
$$

Then the system $K^{*}$, consisting of $1.1,1.2,2.1,2.6,2.7$ and 2.8 (with the atomic predicates $E, Q, P$ and $P^{\prime}$ ), is easily proved to be model-complete

(9) This will become apparent below.

(10) In ordinary language, $P(x)$ means: " $x$ is the first element of the set."

(ii) Note that this step of the proof would fail if we had not adjoined the relation $P(x)$. This is why it is impossible to prove the model-completeness of the original system $K$. 
and complete, whence the completeness of the system 1.1, 1.2, 2.1, $2.7 \mathrm{im}$ mediately follows.

III. We now proceed to prove similar theorems for discretely ordered sets. Such a set can be defined as a model of a system of axioms consisting of Axioms 1.1 and 1.2, combined with

$$
\begin{aligned}
& (x)\{(y) Q(y, x) \vee(\exists y)[T(x, y) \wedge \sim Q(y, x)]\}, \\
& (x)\{(y) Q(x, y) \vee(\exists y)[T(y, x) \wedge \sim Q(x, y)]\},
\end{aligned}
$$

where the relation $T(x, y)$ (read: "if $x<y$, then $y$ is the successor of $x$ ") is defined by the additional axiom $\left({ }^{12}\right)\left({ }^{13}\right)$ :

$$
T(x, y) \cdot \equiv \cdot(z)[Q(z, x) \vee Q(y, z)] .
$$

This system becomes complete if Axiom 2.2 or 2.4 is added:

2.12. THEOREM. The system of axioms for a discretely ordered set with no first and no last element in it (i.e., the system 1.1, 1.2, 2.9, 2.10, 2.11, 2.2) is complete. So is also the system of axioms for a discretely ordered set with a first but no last element in it (i.e. 1.1, 1.2, 2.9, 2.10, 2.11, 2.4).

Proof. Let $K$ be the system mentioned in the first part of the theorem (with the atomic predicates $E, Q, T$ ). Further, let $M$ be a model of $K, M^{\prime}$ its extension, and $Y$ a primitive statement defined in $M . Y$ means this time (see 1.4) that a particular system of conditions of the types

$$
\alpha=\beta, \alpha \neq \beta, \alpha<\beta, \alpha \nless \beta, T(\alpha, \beta), \sim T(\alpha, \beta)
$$

is satisfiable. Assuming that 2.12 .1 is satisfiable in $M^{\prime}$, we shall prove the same for $M$ (this will establish the model-completeness of $K$ ). First we note that every condition of the type $\sim T(\alpha, \beta)$ can be represented as $(\exists z)[\sim Q(\beta, z) \wedge \sim Q(z, \alpha)]$ (see 2.11) and thus be replaced by two inequalities, $z<\beta$ and $\alpha<z$ [where $z$ is to be treated as a new "unknown" in the system 2.12.1]. Furthermore, we can eliminate from 2.12.1 all formulae of the types $\alpha \neq \beta, \alpha \nless \beta$, and $\alpha=\beta$ in the same way as in the proof of 2.3. After these simplifications, 2.12.1 reduces to a finite number of conditions of the two types

$$
\alpha<\beta, \quad T(\alpha, \beta)
$$

only. Our task then reduces to proving that 2.12.2 is satisfiable in $M$, assuming that it is satisfiable in $M^{\prime}$.

We shall say that an $m$-tuple of elements, $\left(x_{1}, x_{2}, \cdots, x_{m}\right)$, is a "chain" if every $x_{i}$ is the successor of $x_{i-1}$, i.e. if $\sim Q\left(x_{i}, x_{i-1}\right) \wedge T\left(x_{i-1}, x_{i}\right), i=2$,

(12) It is more convenient to retain $T$ as a new atomic predicate than to eliminate it (and Axiom 2.11) by substituting the value of $T$ from 2.11 in 2.9 and 2.10 .

(13) By "successor" we mean "immediate successor." 
$\cdots, m$. From the fact that the predicates $Q$ and $T$ are atomic and that $M^{\prime}$ is an extension of $M$ it then follows that any $m$-tuple of elements of $M$ is a chain in $M$ if, and only if, it is a chain in $M^{\prime}$ as well. It is also easily seen that, if $\left(x_{1}, x_{2}, \cdots, x_{m}\right)$ is a chain in $M^{\prime}$ and one of the $x_{i}$ belongs to $M$, then so do all the elements $x_{i}$ of the chain.

Now let $\left(y_{1}, y_{2}, \cdots, y_{n}\right)$ be a solution of 2.12 .2 in $M^{\prime}$, and let $a_{j}(j=1,2, \cdots, m)$ be the constants of $M$ occurring in 2.12.2. As in the proof of 2.3 , we may assume that the $a_{j}$ and $y_{i}$ are arranged in one ascending sequence, say 2.3.2; and it is our task to replace the $y_{i}$ by suitable elements of $M$, so that the inequalities 2.3 .2 remain preserved and also all relations of type $T(\alpha, \beta)$ remain valid as far as they are postulated in 2.12.2. As regards the replacement of the $y_{i}$ which precede all the $a_{j}$ or follow them in 2.3.2, it is easy to meet these requirements, because $M$ has, by assumption, no first and no last element. So we have only to consider the cases when some of the $y_{i}$ lie between $a_{j}$ and $a_{j+1}$, say

$$
a_{j}<y_{k}<y_{k+1}<\cdots<y_{k+m}<a_{j+1} .
$$

There are two possibilities:

(I) $a_{j}$ and $a_{j+1}$ are endpoints of a chain. Then, by what has been said above, all elements of the chain (in particular, $y_{k}, y_{k+1}, \cdots, y_{k+m}$ ) must belong to $M$ (for so do $a_{j}$ and $a_{j+1}$ ), so that we need not replace the $y_{i}$ at all.

(II) $a_{j}$ and $a_{j+1}$ are connected by no chain. As is easily seen, this means that between $a_{j}$ and $a_{j+1}$ there are infinitely many elements of $M$. But then there is certainly no difficulty in selecting from the interval $\left(a_{j}, a_{j+1}\right)$ any finite number of elements of $M$, in replacement of the $y_{i}$ in 2.12.3. It is also easy to select them in such a way that some of them are successors of others, whenever such relations hold between the corresponding $y_{i}$, so that the required conditions of the type $T(\alpha, \beta)$ will also be satisfied.

Thus we see that the system 2.12 .2 is satisfiable in $M$ whenever it is satisfiable in $M^{\prime}$. In other words, $K$ is model-complete, by 1.6. On the other hand, the system $K$ has prime models (e.g. the ordered set of all integers). Therefore $K$ is also complete in the ordinary sense, and this completes the proof of the first part of Theorem 2.12.

The second part of 2.12 differs from the first one in that the system of axioms $1.1,1.2,2.9,2.10,2.11,2.4$ is not model-complete. It is, however, easily shown that it becomes model-complete if, as in 2.5 , the new atomic predicate $P$ and Axiom 2.6 are adjoined. The rest of the proof then runs on the same lines as in the first part of the theorem.

The case of a discretely ordered set with a last but no first element is treated in exactly the same way as above.

Finally, we take up the system of Axioms 1.1, 1.2, 2.9, 2.10, 2.11, 2.7, for a discretely ordered set with both a last and a first element in it. This system is incomplete for it does not state whether or not the set is finite. We shall 
show that it becomes complete if the following sequence of axioms (which exclude the finite case) is added:

$$
\begin{aligned}
\left(\exists x_{1}\right)\left(\exists x_{2}\right) & \cdots\left(\exists x_{n}\right)\left[\sim E\left(x_{1}, x_{2}\right) \wedge \sim E\left(x_{1}, x_{3}\right) \wedge \cdots\right. \\
& \wedge \sim E\left(x_{1}, x_{n}\right) \wedge E\left(x_{2}, x_{3}\right) \wedge \cdots \\
& \left.\wedge \sim E\left(x_{n-1}, x_{n}\right)\right], \quad n \sim E\left(x_{2}, x_{n}\right) \wedge \cdots
\end{aligned}
$$

2.14. THEOREM. The system of axioms for an infinite discretely ordered set with a first and a last element in it (i.e. the system 1.1, 1.2, 2.9, 2.10, 2.11, 2.7, 2.13) is complete.

Proof. This system (call it $K$ ) is transformed into a model-complete system $K^{*}$ by adjoining the atomic predicates $P$ and $P^{\prime}$, and the Axioms 2.6 and 2.8. This is proved in the same way as in 2.12, with the deviation that the system 2.12.1 now contains also formulae of the types $P(\alpha), \sim P(\alpha)$, $P^{\prime}(\alpha)$ and $\sim P^{\prime}(\alpha)$. The completeness of $K^{*}$ then is derived from the existence of prime models, e.g., the ordered set of all positive integers (in natural order) followed by all negative integers in the reverse order:

$$
1,2,3, \cdots, n, \cdots,-n, \cdots,-3,-2,-1
$$

(in other words, the prime model is an ordered set of type $\omega+\omega^{*}$ where $\omega$ is the order type of the natural series).

The results of this section can be summed up in one theorem as follows:

2.15. THEOREM. Two densely ordered sets (and, similarly, two infinite discretely ordered sets) are elementarily equivalent if, and only if, each of them, or none of them, has a first element, and each of them, or none of them, has a last element.

In fact, the completeness of the systems of axioms analyzed above, obviously, implies that any two models of such a system must have all their elementary properties in common, as all such properties must be deducible from the system of axioms in question.

If $\eta$ and $\omega$ denote the order types of the set of all rationals and the set of all positive integers respectively (both sets in their natural order), we may also say that there are only four elementarily distinct types of densely ordered sets, viz.

$$
\eta, \quad \eta+1, \quad 1+\eta, \quad 1+\eta+1,
$$

and only four elementarily distinct types of infinite discretely ordered sets, to wit:

$$
\omega, \quad \omega^{*}, \quad \omega+\omega^{*}, \quad \omega^{*}+\omega
$$

(here $\omega^{*}$ denotes the order type reverse to $\omega$ ). 
In this way a complete classification of all densely ordered sets and all infinite discretely ordered sets has been achieved.

Now we shall take up the study of ordered groups.

3. Some algebraic properties of abelian groups. We shall need several concepts and propositions referring to ordered abelian groups:

3.1. Definition. Given an abelian group $M$ and a positive integer $p$, we define the pth congruence invariant of $M$ (denoted by " $[p] M$ " or, briefly, by " $[p]$ ") to be the maximum finite number of elements in $M$ which are mutually incongruent modulo $p\left({ }^{14}\right)$. If such a finite number does not exist, we put $[p]=\infty$, without distinguishing between infinities of different cardinalities. The symbol $\infty$ will be treated as a positive number, with the usual conventions as to the arithmetical operations and inequalities. [p] will be called a prime invariant of $M$ if $p$ is a prime.

3.2. Definition. By a linear system we mean any finite system of equations, inequalities, congruences and (or) incongruences of the form

$$
\begin{array}{lr}
\sum_{j=1}^{n} q_{i j} x_{j}=a_{i}, & \left(i=1,2, \cdots, m_{1}\right) ; \\
\sum_{j=1}^{n} q_{i j} x_{j}<a_{i}, & \left(i=m_{1}+1, m_{1}+2, \cdots, m_{2}\right) ; \\
\sum_{j=1}^{n} q_{i j} x_{j} \equiv a_{i}\left(\bmod r_{i}\right), & \left(i=m_{2}+1, m_{2}+2, \cdots, m_{3}\right) ; \\
\sum_{j=1}^{n} q_{i j} x_{j} \not \equiv a_{i}\left(\bmod r_{i}\right), & \left(i=m_{3}+1, m_{3}+2, \cdots, m_{4}\right),
\end{array}
$$

where the $q_{i j}$ are given integers, the $x_{j}$ are unknowns, the $r_{i}$ are positive integers, and the $a_{i}$ are given elements of an ordered abelian group $M$. The $a_{i}$ will be called the "constants" of the system.

3.3. Definition. An ordered abelian group $M$ is said to be

(i) discretely ordered if it contains a smallest positive element (this element is called the unit of $M$ ).

(ii) densely ordered if it has no unit.

(iii) regularly discrete if $M$ is discretely ordered and such that $[p] M=p$ for every prime $p$.

(iv) regularly dense if, for any positive integer $p$ and any elements $a, b \in M(a<b)$, there is an element $x \in M(a<x<b)$ which is divisible by $p$.

(v) regularly ordered if $M$ is regularly discrete or regularly dense.

(vi) archimedean if, for any positive elements $x, y \in M$, there is a positive integer $n$ with $n x>y$.

${ }^{(14)}$ As usual, we say that two elements $x, y \in M$ are congruent modulo $p$ ( $p$ being a positive integer), and write $x \equiv y(\bmod p)$ if there exists an element $z \in M$ such that $x=y+p z$. In particular, $x \equiv 0(\bmod p)$ means that $x$ is divisible by $p$. 
The following propositions can be proved $\left({ }^{15}\right)$ :

3.4. THEOREM. Every discretely ordered archimedean group is regularly discrete. Every densely ordered archimedean group is regularly dense $\left({ }^{16}\right)$.

3.5. THEOREM. Let $p_{1}, p_{2}, \cdots, p_{n}, \cdots$ be the ascending sequence of all primes, and $m_{1}, m_{2}, \cdots, m_{n}, \cdots$ an arbitrary sequence where each $m_{n}$ is either a non-negative integer or $\infty$. Then there always exists a densely ordered archimedean group whose prime invariants, $\left[p_{n}\right]$, are given by

$$
\left[p_{n}\right]=\left(p_{n}\right)^{m_{n}}, \quad n=1,2,3, \cdots
$$

3.6. THEOREM. In every ordered abelian group the sequence of its prime invariants $\left[p_{n}\right]$ is as indicated in 3.5.1, i.e. every prime invariant is a (possibly infinite) power of the corresponding prime.

3.7. THEOREM. Let $M$ and $M^{\prime}\left(M \subseteq M^{\prime}\right)$ be two regularly discrete groups having one and the same unit [see 3.3 (i)] and such that $M$ is a serving subgroup of $M^{\prime}{ }^{(17)}$. Then every linear system [see 3.2] which has a solution in $M^{\prime}$ can also be solved in $M$, provided only that its constants $a_{i}$ all are elements of $M$.

The same theorem holds also for any two regularly dense groups, $M$ and $M^{\prime}$, with the modification that, instead of having the same unit, the two groups must have the same prime invariants, i.e. $[p] M=[p] M^{\prime}$, for every prime $p$.

3.8. Theorem. Let $A$ and $B$ be two countable disjoint regularly dense groups having the same prime invariants. Then there exists a regularly dense group $M$ such that: (a) $A$ and $B$ are serving subgroups of $M$; (b) $M$ has the same prime invariants as $A$ and $B\left({ }^{18}\right)$.

REMARK. It is useful to note that any formula of the form 3.5.1 is elementary. In fact, the formula $[p]=q$, where $p, q$ are positive integers, can be

(15) These propositions will be formulated here without proof, in the form of an announcement only. The proofs will be given in a separate article [11] to be published by one of the present authors, so as not to overload this paper (which is primarily metamathematical) with purely algebraic arguments.

(16) The converse statements are not true as can be easily proved by examples. For instance, the additive group of all complex numbers, ordered lexicographically, is regularly ordered but not archimedean. Thus the class of all regularly ordered groups is larger than that of all archimedean groups.

(17) "Serving subgroup" in the sense of Prüfer's "Servanzuntergruppe" [6], or Kurosh's "serving subgroup" [4]. Kaplansky [3] and Braconnier [1] use instead the term "pure subgroup." Note that the subgroup of an ordered group is supposed to be ordered in the same way as the containing group.

(18) The zero-elements of $A$ and $B$ must, of course, be "identified" when they are embedded in $M$. In our terminology, however, it suffices that their zeros become "equivalent" in the sense of our axioms 1.1. As the relation $E$ need not coincide with logical identity, the groups $A$ and $B$ may still be considered as fully disjoint (the zero-elements of $A$ and $B$ are "equivalent" in $M$, but not logically identical). 
written as the conjunction of the following two formulae (in which all the congruences and incongruences are modulo $p)\left({ }^{19}\right)$ :

$$
\begin{aligned}
&\left(\exists x_{1}\right)\left(\exists x_{2}\right) \cdots\left(\exists x_{q}\right)(y)\left[y \equiv x_{1} \vee y \equiv x_{2} \vee \cdots \vee\right.\left.\cdots x_{q}\right] \\
&\left(\exists x_{1}\right) \cdots\left(\exists x_{q}\right)\left[x_{1} \not \equiv x_{2} \wedge x_{1} \not \equiv x_{3} \wedge \cdots \wedge x_{1} \neq x_{q} \wedge \cdots\right. \\
&\left.\wedge x_{q-1} \neq x_{q}\right]
\end{aligned}
$$

On the other hand, every congruence, say $y \equiv x(\bmod n)$ is an elementary formula, for it can be rendered as

$$
(\exists z)\left[D_{n}(z) \wedge S(z, y, x)\right]
$$

where $D_{n}$ is defined as in 1.5. Thus it suffices to replace in 3.9 and $3.9^{\circ}$ every formula of the form " $y \equiv x_{i}$ " or " $y \neq x_{i}$ " by its explicit expression in order to obtain the elementary representation of " $[p]=q$," with a finite $q$. If, however, $q=\infty$, we replace 3.9 and $3.9^{\circ}$ by an infinite sequence of elementary formulas, viz.:

$$
\begin{array}{r}
\left(x_{1}\right)\left(x_{2}\right) \cdots\left(x_{m}\right)(\exists y)\left[y \not \equiv x_{1} \wedge y \not \equiv x_{2} \wedge \cdots \wedge y \not \equiv x_{m}\right], \\
m=1,2,3, \cdots,
\end{array}
$$

where again each congruence and incongruence is to be expressed in terms of the atomic predicates, as shown above. The sequence of formulae 3.5.1, which specifies the prime invariants of a given group, can then always be written as a countable collection of elementary formulae, in terms of the predicates $E, Q$ and $S$. Together with our basic axioms, 1.1, 1.2 and 1.3, such a sequence of formulae can be treated as a system of axioms, which we shall call a system of axioms for an ordered abelian group with specified prime invariants $\left({ }^{20}\right)$. Theorems 3.5 and 3.6 can now be interpreted as a statement of sufficient and necessary conditions for such a system to be consistent, and can be reformulated as follows:

3.12. Theorem. A system of axioms for an ordered abelian group with specified prime invariants is consistent if, and only if, in all axioms of the form 3.9 and $3.9^{\circ}$ contained in the system, the positive integer $q$ is a power of the corresponding prime $p$ (the exponent of the power being a non-negative integer) ${ }^{(21}$ ).

4. Classification of regularly ordered groups. We are now able to tackle our main problem. Our starting point will be the system of Axioms 1.1, 1.2, 1.3 which we shall briefly call "1.1-1.3." We shall also consider the (counta-

(19) Formula 3.9 expresses the fact that there are not more than $q$ elements incongruent modulo $p$, while $3.9^{\circ}$ states that there are at least $q$ such elements. Combined, they state that $[p]=q$.

${ }^{(20)}$ Of course, there are infinitely many such systems, depending on the choice of the prime invariants.

(21) No restriction is necessary with regard to axioms of the form 3.11. 
ble) system arising from 1.1-1.3 through the adjunction of the relations $D_{n}(x)$ and the Axioms 1.5 , for $n=2,3,4, \ldots$. In this extended system, the atomic predicates are $E, Q, S$ and all the $D_{n}$; it will be referred to as "1.11.5." ${ }^{(22)}$.

I. First we shall analyze discretely ordered groups. Such a group can be regarded as a model of a system of axioms consisting of 1.1-1.3 (or 1.1-1.5) and the following two additional axioms introducing a new atomic predicate $U\left({ }^{23}\right)$ :

$$
\begin{aligned}
& (\exists x) U(x) . \\
& U(x) \cdot \equiv \cdot(\exists y)\{S(y, y, y) \wedge \sim Q(x, y) \wedge(z)[Q(z, y) \vee Q(x, z)]\} .
\end{aligned}
$$

In order to obtain the system of axioms for a regularly discrete group [see 3.3 (iii) ], we have to add a sequence of axioms stating that

$$
[p]=p, \quad \text { for every prime } p \text {. }
$$

This system of axioms is elementary (for the axioms 4.3 can be expressed in elementary form, as was shown in the final Remark to §3). It is also consistent, as it has models (e.g., the ordered additive group of all integers). We shall now prove the following:

4.4. TheOREM. The system of axioms for a regularly discrete group (consisting of Axioms 1.1-1.3, 4.1, 4.2 and 4.3) is complete.

Proof. Let $K$ be the system of axioms in question, and let $K^{*}$ be the system arising from it through the adjunction of the relations $D_{n}(x)$ and axioms $1.5 ;$ i.e., $K^{*}$ consists of $1.1-1.5,4.1,4.2,4.3$; and its atomic predicates are $E, Q, S, U$ and all the $D_{n}$. We shall show that $K^{*}$ is model-complete. Let then $M$ be a model of $K^{*}, M^{\prime}$ its extension, and $Y$ a primitive statement defined in $M$ in terms of the atomic predicates $E, Q, S, U$ and $D_{n}$. In ordinary notation, $Y$ states that a finite system of conditions of the types

$$
\begin{aligned}
& \alpha=\beta, \quad \alpha+\beta=\gamma, \quad \alpha<\beta, \quad U(\alpha), \quad \alpha \equiv 0(\bmod n), \\
& \alpha \neq \beta, \quad \alpha+\beta \neq \gamma, \quad \alpha \nless \beta, \quad \sim U(\alpha), \quad \alpha \neq \equiv(\bmod ) n,
\end{aligned}
$$

is satisfiable. Here the formulae of the type $U(\alpha)$ and $\sim U(\alpha)$ can be replaced by $\alpha=1$ and $\alpha \neq 1$ respectively, where 1 denotes the unit of $M\left({ }^{24}\right)$.

(22) Noteworthy is the change caused by the adjunction of the relations $D_{n}(x)$, with respect to model extensions (see $\$ 1$ ). The statement that $M$ is a model of $1.1-1.3$, and $M^{\prime}$ its extension, simply means that $M$ is a subgroup of an ordered abelian group $M^{\prime}$. If, however, $1.1-1.3$ is replaced by $1.1-1.5$, the same statement means that $M$ is a serving subgroup of the ordered abelian group $M^{\prime}$. [For now all the formulae $D_{n}(x)$ are atomic; so whenever $D_{n}(x)$ holds in $M^{\prime}$, it must also hold in $M$, and vice versa.]

(23) The predicate $U$ and Axiom 4.2 could be eliminated by substituting the value of $U(x)$ from 4.2 in 4.1 . It is, however, convenient to retain $U$ as an atomic predicate. In ordinary language, $U(x)$ means: " $x$ is the unit of the group."

(24) The same element 1 is the unit of $M^{\prime}$ as well. This follows easily from the fact that $U(1)$ is an atomic formula, and that $M^{\prime}$ is an extension of $M$. 
Furthermore, as in the proof of 2.3 , we can simplify the system 4.4 .1 by eliminating all inequalities of the type $\alpha \neq \beta, \alpha \nless \beta$ and $\alpha+\beta \neq \gamma$. After these simplifications we shall be left with a system containing only linear equations, congruences, incongruences and inequalities of the type $\alpha<\beta$; which is a special case of what we have defined as a linear system (see 3.2), with its constants in $M\left({ }^{25}\right)$. By 1.6, we have to show that this system has a solution in $M$ assuming that it is satisfiable in $M^{\prime}$. But this fact immediately follows from Theorem 3.7 if we take into account that $M$ and $M^{\prime}$, being models of $K^{*}$, are regularly discrete groups, have the same unit (see footnote) and, furthermore, $M$ is a serving subgroup of $M^{\prime}$ [this is a consequence of the adjunction of the relations $D_{n}(x)$; see footnote 22]. This completes the proof of the modelcompleteness of $K^{*}$. On the other hand, as is easily seen, the ordered additive group of all integers is a prime model of $K^{*}$ [for in every regularly discrete group there is a serving subgroup isomorphic to the group of integers, to wit: the subgroup of all integral multiples of the unit]. Hence, by $1.7, K^{*}$ is also complete in the ordinary sense, which implies the completeness of $K$ as well. Thus the theorem is proved.

II. Next we shall consider regularly dense groups. The class of these groups can be characterized by an elementary system of axioms consisting of 1.1-1.5 (with the atomic predicates $E, Q, S$ and $D_{n}$ ), and the following sequence of axioms:

$$
\begin{array}{r}
(x)(y)\left\{Q(y, x) \vee(\exists z)\left[\sim Q(z, x) \wedge \sim Q(y, z) \wedge D_{n}(z)\right]\right\}, \\
n=2,3,4, \cdots .
\end{array}
$$

If, in addition, the prime invariants are specified by adjoining still another sequence of axioms, of the form 3.5.1 (translated into formal language as shown in formulae $3.9,3.9^{\circ}$ and 3.11 ), then a new elementary system of axioms (with the same atomic predicates) is obtained which we shall call a system of axioms for a regularly dense group with specified prime invariants $\left({ }^{26}\right)$. Such a system is consistent if, and only if, it satisfies the requirements of Theorem 3.12. We shall now prove that, if consistent, it is also complete.

4.6. THEOREM. Let $K$ be a consistent system of axioms for a regularly dense group with specified prime invariants. Then $K$ is complete.

Proof. Exactly as in 4.4 we show that $K$ is model-complete (the proof is even simpler due to the absence of the predicate $U$ ). As regards the completeness of $K$, we apply in this case not 1.7 but 1.8 and 3.8. Theorem 3.8, when translated into our formal terminology, states that any two disjoint countable models of $K$ can be embedded in a joint extension. On the other hand, $K$ is obviously a countable system of axioms with no constants, and with infinite models only. Hence, by 1.8 , the completeness of $K$ follows, and our theorem is proved.

(25) The constants helong to $M$, for $Y$ has been supposed to be defined in $M$ (see $\$ 1$ ).

${ }^{(26)}$ Footnote 20 applies to this system too. The predicates $D_{n}$ and Axioms 1.5 could, of course, be eliminated, but it is more convenient to leave them in the system. 
Theorems 4.4 and 4.6 can be summed up as follows:

4.7. Theorem. All regularly discrete groups are elementarily equivalent. Two regularly dense groups, $A$ and $B$, are elementarily equivalent if, and only if, they have the same prime invariants, i.e. if $[p] A=[p] B$ for every prime $p$.

This theorem leads to a complete classification of all regularly ordered groups and, among them, all archimedean groups (see 3.4). In this classification all regularly discrete groups form one separate subclass. The class of all regularly dense groups is divided into subclasses each of which consists of groups having the same prime invariants. Thus every specification of the prime invariants by means of a sequence of the form 3.5.1 generates exactly one subclass of regularly dense groups. These subclasses are not empty as each of them contains, by 3.5 , at least one archimedean group. There are as many subclasses as there are sequences of the form 3.5.1, i.e. they form a family of continuum power. The subclasses are clearly disjoint.

Inside each subclass, the groups belonging to it are elementarily equivalent, i.e. they cannot be distinguished from each other by any properties formalizable in the lower predicate calculus. As each subclass contains at least one archimedean group, it follows that every regularly ordered group is elementarily equivalent to some archimedean group. Thus we may say that the concept of a regularly ordered group is the elementary counterpart to that of an archimedean group $\left({ }^{27}\right)$. This throws a new light on the significance of that concept. A more detailed study of regularly ordered groups will be left for a separate paper [11].

\section{REFERENCES}

1. J. Braconnier, Sur les groupes topologiques localement compacts, J. Math. Pures Appl. vol. 27 (1948) pp. 1-85.

2. F. Hausdorff, Set theory, 3rd ed., New York, 1957.

3. I. Kaplansky, Infinite Abelian groups, University of Michigan Press, 1954.

4. A. G. Kurosh, The theory of groups, New York, 1956.

5. C. H. Langford, Some theorems on deducibility, Ann. of Math., 2nd ser., vol. 28 (19261927) pp. 16-40.

6. H. Prüfer, Untersuchungen über die Zerlegbarkeit der abzählbaren primären abelschen Gruppen, Math. Z. vol. 17 (1923) pp. 35-61.

7. A. Robinson, Ordered structures and related concepts, mathematical interpretation of formal systems, Studies in Logic and the Foundations of Mathematics, Amsterdam, 1954, pp. 51-56.

8. A. Robinson, Complete theories, Amsterdam, North-Holland Publishing Co., 1956.

9. W. Szmielew, Elementary properties of Abelian groups, Fund. Math. vol. 41(2) (1955) pp. $203-271$.

10. A. Tarski, Grundzüge des Systemenkalküls, Fund. Math. vol. 25 (1935) pp. 503-526 and vol. 26 (1936) pp. 283-301.

11. E. Zakon, Generalized archimedian groups, submitted for publication in Trans. Amer. Math. Soc.

HEBREW UNIVERSITY, JERUSALEM, ISRAEL

Essex College, Assumption University of Windsor, Windsor, Ontario, Canada

(27) The concept of an archimedean group itself cannot be formalized in the lower predicate calculus. 